\title{
Vacuum energy in Einstein-Gauss-Bonnet anti-de Sitter gravity
}

\author{
Georgios Kofinas ${ }^{1}$ and Rodrigo Olea ${ }^{2}$ \\ ${ }^{1}$ Departament de Física Fonamental, Universitat de Barcelona \\ Diagonal 647, 08028 Barcelona, Spain and \\ ${ }^{2}$ Centro Multidisciplinar de Astrofísica - CENTRA, \\ Departamento de Física, Instituto Superior Técnico, \\ Universidade Técnica de Lisboa, Av. Rovisco Pais 1, 1049-001 Lisboa, Portugal
}

(Dated: April 4, 2018)

\begin{abstract}
A finite action principle for Einstein-Gauss-Bonnet anti-de Sitter gravity is achieved supplementing the bulk Lagrangian by a suitable boundary term, whose form substantially differs in odd and even dimensions. For even dimensions, this term is given by the boundary contribution in the Euler theorem with a coupling constant fixed demanding the spacetime to have constant (negative) curvature in the asymptotic region. For odd dimensions, the action is stationary under a boundary condition on the variation of the extrinsic curvature. A well-posed variational principle leads to an appropriate definition of energy and other conserved quantities using the Noether theorem, and to a correct description of black hole thermodynamics. In particular, this procedure assigns a nonzero energy to anti-de Sitter spacetime in all odd dimensions.
\end{abstract}

In recent years, several experiments carried out suggest observational evidence for a positive value of the cosmological constant [1]. Nonetheless, from a theoretical point of view, the idea of existence of extra dimensions and alternative gravity theories does not rule out a negative cosmological constant in a higher-dimensional spacetime. On the contrary, for example, particular braneworld models induce a zero or positive cosmological constant on a four-dimensional Universe [2].

A negative cosmological constant is also appealing because of the possibility of a profound connection between anti-de Sitter gravity and a conformal field theory (CFT) living on its boundary, that has attracted a considerable attention in the literature 3]. Even though some remarkable progress has been achieved on a rather case-by-case basis, a general proof of this duality remains unknown. In that context, the existence of a nonzero energy for anti-de Sitter (AdS) vacuum spacetime in the gravity side may be helpful to identify the corresponding CFT at the boundary. Indeed, in five dimensions, the matching between the zero-point energy for Schwarzschild-AdS black hole and the induced (Casimir) energy of a precise boundary field theory is one of the best known examples that realizes this bulk/boundary correspondence [4]. General Relativity with AdS asymptotics requires a regularization procedure in order to define a finite energy for the solutions of the theory. Logically, a vacuum energy can appear only when this mechanism does not invoke the substraction of a background configuration as, e.g., in Hamiltonian formalism. That is the case of the counterterms method [5, [6], where the finiteness of the action and its energy-momentum tensor is obtained by the addition of covariant functionals of the boundary metric, constructed by solving the Einstein equations in a given asymptotic form of the metric [7]. In spite this algorithm provides the correct counterterms for many cases, in high enough dimension it becomes rather cumbersome, what makes the full series still unknown. It is clear that the inclusion of quadratic curvature terms in the action will turn this method of regularization even more complex.

In this article, we introduce the boundary terms that make the definition of energy in Einstein-Gauss-Bonnet gravity finite in all dimensions. On the contrary to the standard approach, these terms appear as given geometrical structures, where the regularization procedure amounts to fix a single coupling constant in a well-defined variational problem for precise asymptotic conditions.

In $D=d+1$ dimensions, the EGB action can be supplemented by a boundary term $B_{d}$

$$
\begin{aligned}
I_{D}=\frac{1}{16 \pi G_{D}} \int_{M} d^{D} x \sqrt{-\hat{g}}[ & {\left[\hat{R}-2 \Lambda+\alpha\left(\hat{R}_{\mu \nu \kappa \lambda} \hat{R}^{\mu \nu \kappa \lambda}-\right.\right.} \\
& \left.\left.-4 \hat{R}_{\mu \nu} \hat{R}^{\mu \nu}+\hat{R}^{2}\right)\right]+c_{d} \int_{\partial M} B_{d},(1)
\end{aligned}
$$

that regularizes either the conserved quantities and the Euclidean action. Hatted quantities stand for $D$ dimensional ones. It is well known that coupling a GaussBonnet term still gives second-order field equations, and in an effective action of string theory it corresponds to the leading order quantum corrections to standard gravity [8]. Braneworld cosmologies with curvature corrections have also been considered, e.g. in 9, 10]. For later convenience, we take the above action in the language of differential forms

$$
\begin{aligned}
& I_{D}=\frac{1}{16 \pi(D-2) ! G_{D}} \int_{M} \epsilon_{A_{1} \ldots A_{D}}\left(\hat{\mathcal{R}}^{A_{1} A_{2}}+\frac{D-2}{D \ell^{2}} e^{A_{1}} e^{A_{2}}\right) e^{A_{3}} \ldots e^{A_{D}} \\
& +\alpha(D-2)(D-3) \epsilon_{A_{1} \ldots A_{D}} \hat{\mathcal{R}}^{A_{1} A_{2}} \hat{\mathcal{R}}^{A_{3} A_{4}} e^{A_{5}} \ldots e^{A_{D}}+c_{d} \int_{\partial M} B_{d},(2)
\end{aligned}
$$

where the cosmological constant $\Lambda=-\frac{(D-1)(D-2)}{2 \ell^{2}}$, the orthonormal vielbein $e^{A}=e_{\mu}^{A} d x^{\mu}$ and the curvature 2form is defined as $\hat{\mathcal{R}}^{A B}=\frac{1}{2} \hat{R}_{\kappa \lambda}^{\mu \nu} e^{A}{ }_{\mu} e^{B}{ }_{\nu} d x^{\kappa} d x^{\lambda}$ in terms of the spacetime Riemman tensor. Wedge products are omitted throughout. We will also use the symbol 
$\kappa_{D}=\left[16 \pi(D-2) ! G_{D}\right]^{-1}$. We will consider spaces of negative constant curvature in the asymptotic region, which means that at the boundary

$$
\hat{R}_{\kappa \lambda}^{\mu \nu}+\frac{1}{\ell_{e f f}^{2}} \delta_{[\kappa \lambda]}^{[\mu \nu]}=0
$$

where the effective AdS radius is $\frac{1}{\ell_{\text {eff }}^{2}}=\frac{1 \pm \sqrt{1+8 \Lambda \alpha^{*}}}{2(D-1)(D-2) \alpha^{*}}$ and $\alpha^{*}=\alpha \frac{(D-3)(D-4)}{(D-1)(D-2)}$.

In general, any spacetime metric can be put in Gaussnormal coordinates $d s^{2}=N^{2}(\rho) d \rho^{2}+h_{i j}(x, \rho) d x^{i} d x^{j}$. For AAdS spaces in EGB gravity (3), the FeffermanGraham expansion of the metric is also valid and defined by $N=\ell_{\text {eff }} / 2 \rho, h_{i j}=g_{i j}(x, \rho) / \rho$ and the expansion $g_{i j}=g_{(0) i j}+\rho g_{(1) i j}+\rho^{2} g_{(2) i j}+\ldots$, where $g_{(0)}$ represents the metric of the conformal boundary $\rho=0$ [11, 12]. The extrinsic curvature $K_{i j}=-\partial_{\rho} h_{i j} / 2 N$ takes the form

$$
K_{j}^{i}=K_{j \ell} h^{\ell i}=\frac{1}{\ell_{e f f}} \delta_{j}^{i}-\frac{\rho}{\ell_{e f f}}\left(g_{(0)}^{-1} g_{(1)}\right)_{j}^{i}+\ldots
$$

that means that at the boundary

$$
K_{j}^{i}=\frac{1}{\ell_{e f f}} \delta_{j}^{i}
$$

In the variational problem of a gravity theory $h_{i j}$ and $K_{i j}$ are independent variables. A well-posed action principle is defined for suitable boundary conditions for these fields that makes the on-shell action stationary. In the present paper, for the odd-dimensional case, we will consider that at the boundary the variations obey

$$
\delta K_{j}^{i}=0
$$

-what is compatible with fixing the conformal metric on $\partial M[13]-$ and introduce the appropriate boundary term which makes the EGB action to reach an extremum. Contrary to the standard Dirichlet approach to regularize the AdS action, where the counterterms are constructed out only of intrinsic quantities, the boundary terms proposed here explicitly contain also the extrinsic curvature, making evident that we are not dealing with the same action principle.

In AdS gravity, odd and even dimensions have different essential features (existence of Weyl anomalies, vacuum energy, etc.) that suggest technical differences concerning their regularization (e.g., as in the standard holographic renormalization). As we shall see below, the difference in the prescriptions to treat even and odd dimensional cases is related to the existence of topological invariants 14,15$]$.

Odd-dimensional case. Starting for conciseness with $D=5$, the boundary term that leads to a welldefined variation of the action is

$$
\begin{aligned}
B_{4} & =\epsilon_{A_{1} . . A_{5}} \theta^{A_{1} A_{2}} e^{A_{3}}\left(\mathcal{R}^{A_{4} A_{5}}+\frac{1}{2} \theta_{C}^{A_{4}} \theta^{C A_{5}}+\frac{1}{6 \ell_{e f f}^{2}} e^{A_{4}} e^{A_{5}}\right) \\
& =\sqrt{-h} \delta_{\left[j_{1} j_{2} j_{3}\right]}^{\left[i_{1} i_{2} i_{3}\right]} K_{i_{1}}^{j_{1}}\left(R_{i_{2} i_{3}}^{j_{2} j_{3}}-K_{i_{2}}^{j_{2}} K_{i_{3}}^{j_{3}}+\frac{1}{3 \ell_{e f f}^{2}} \delta_{i_{2}}^{j_{2}} \delta_{i_{3}}^{j_{3}}\right) d^{4} x,
\end{aligned}
$$

where the second fundamental form $\theta^{A B}=n^{A} K^{B}-$ $n^{B} K^{A}, K^{A}=K_{B}^{A} e^{B}, K_{A B}=-h_{A}^{C} h_{B}^{D} n_{C ; D}$, and $n^{A}$ is the unit normal vector at the boundary. In the ordered frame $\left(e^{1}=N d \rho, e^{a}=e_{i}^{a} d x^{i}\right)$ its only non-vanishing components are $\theta^{1 a}=-K^{a}=-K_{j}^{i} e_{i}^{a} d x^{j} . \mathcal{R}^{A B}$ and $R_{k \ell}^{i j}$ are the curvature 2 -form and the Riemann tensor of the boundary metric respectively. An arbitrary variation of the action (2) produces the equations of motion plus a surface term

$$
\begin{aligned}
& \delta I_{5}=\int_{M}(\text { E.O.M. })+\int_{\partial M} 2 \kappa_{5} \epsilon_{a b c d} \delta K^{a} e^{b}\left(e^{c} e^{d}+12 \alpha \hat{\mathcal{R}}^{c d}\right) \\
& \quad+4 c_{4} \epsilon_{a b c d} \delta K^{a} e^{b}\left(\hat{\mathcal{R}}^{c d}+\frac{1}{3 \ell_{\text {eff }}^{2}} e^{c} e^{d}\right) \\
& -2 c_{4} \epsilon_{a b c d}\left(\delta K^{a} e^{b}-K^{a} \delta e^{b}\right)\left(\mathcal{R}^{c d}-\frac{1}{2} K^{c} K^{d}+\frac{1}{2 \ell_{e f f}^{2}} e^{c} e^{d}\right) \cdot(7)
\end{aligned}
$$

The last term in (7) is canceled by the conditions (5), (6). Assuming the asymptotic behavior (3) for the curvature, the coupling $c_{4}$ is fixed as $c_{4}=\left(\ell_{\text {eff }}^{2}-12 \alpha\right) / 128 \pi G_{5}$ to cancel the rest of the terms.

For any odd dimension $D=2 n+1$, the generalization of the previous boundary term can be written in a compact form using parametric integrations

$$
\begin{aligned}
& B_{2 n}=n \int_{0}^{1} d t \int_{0}^{t} d s \epsilon_{A_{1} \ldots A_{2 n+1}} \theta^{A_{1} A_{2}} e^{A_{3}}\left(\mathcal{R}^{A_{4} A_{5}}+t^{2} \theta_{C}^{A_{4}} \theta^{C A_{5}}+\frac{s^{2}}{\ell_{\text {eff }}^{2}} e^{A_{4}} e^{A_{5}}\right) \\
& \ldots\left(\mathcal{R}^{A_{2 n} A_{2 n+1}}+t^{2} \theta_{F}^{A_{2 n}} \theta^{F A_{2 n+1}}+\frac{s^{2}}{\ell_{\text {eff }}^{2}} e^{A_{2 n}} e^{A_{2 n+1}}\right) \\
& =2 n \sqrt{-h} \int_{0}^{1} d t \int_{0}^{t} d s \delta_{\left[j_{1} \ldots j_{2 n-1}\right]}^{\left[i_{1} . i_{2 n-1}\right]} K_{i_{1}}^{j_{1}}\left(\frac{1}{2} R_{i_{2} i_{3}}^{j_{2} j_{3}}-t^{2} K_{i_{2}}^{j_{2}} K_{i_{3}}^{j_{3}}+\frac{s^{2}}{\ell_{e f f}^{2}} \delta_{i_{2}}^{j_{2}} \delta_{i_{3}}^{j_{3}}\right) \\
& \ldots\left(\frac{1}{2} R_{i_{2 n-2} i_{2 n-1}}^{j_{2 n-2} j_{2 n-1}}-t^{2} K_{i_{2 n-2}}^{j_{2 n-2}} K_{i_{2 n-1}}^{j_{2 n-1}}+\frac{s^{2}}{\ell_{e f f}^{2}} \delta_{i_{2 n-2}}^{j_{2 n-2}} \delta_{i_{2 n-1}}^{j_{2 n-1}}\right) d^{2 n} x .
\end{aligned}
$$

The on-shell variation of the action (2) is

$$
\begin{aligned}
& \delta I_{2 n+1}=\int_{\partial M} 2 \kappa_{D} \epsilon_{a_{1} \ldots a_{2 n}} \delta K^{a_{1}}\left(e^{a_{2}} e^{a_{3}}+2 \alpha(D-2)(D-3) \hat{\mathcal{R}}^{a_{2} a_{3}}\right) e^{a_{4}} \ldots e^{a_{2 n}} \\
& +2 n c_{2 n} \int_{0}^{1} d t \epsilon_{a_{1} \ldots a_{2 n}} \delta K^{a_{1}} e^{a_{2}}\left(\hat{\mathcal{R}}^{a_{3} a_{4}}+\frac{t^{2}}{\ell_{e f f}^{2}} e^{a_{3}} e^{a_{4}}\right) \ldots\left(\hat{\mathcal{R}}^{a_{2 n-1} a_{2 n}}+\frac{t^{2}}{\ell_{e f f}^{2}} e^{a_{2 n-1}} e^{a_{2 n}}\right) \\
& -2 n c_{2 n} \int_{0}^{1} d t t \epsilon_{a_{1} . . a_{2 n}}\left(\delta K^{a_{1}} e^{a_{2}}-K^{a_{1}} \delta e^{a_{2}}\right)\left(\mathcal{R}^{a_{3} a_{4}}-t^{2} K^{a_{3}} K^{a_{4}}+\frac{t^{2}}{\ell_{e f f}^{2}} e^{a_{3}} e^{a_{4}}\right) \\
& \quad \ldots\left(\mathcal{R}^{a_{2 n-1} a_{2 n}}-t^{2} K^{a_{2 n-1}} K^{a_{2 n}}+\frac{t^{2}}{\ell_{e f f}^{2}} e^{a_{2 n-1}} e^{a_{2 n}}\right),
\end{aligned}
$$

and vanishes for the same boundary conditions as in the five-dimensional case when

$$
c_{2 n}=-\ell_{\text {eff }}^{2 n-2} \frac{\kappa_{D}}{n}\left[1-\frac{2 \alpha}{\ell_{\text {eff }}^{2}}(D-2)(D-3)\right]\left[\int_{0}^{1} d t\left(t^{2}-1\right)^{n-1}\right]^{-1} .
$$

Conserved quantities and vacuum energy. The charges $Q(\xi)$ associated to asymptotic Killing vectors $\xi$ -computed using the Noether theorem- are defined as integrals on the boundary of the spatial section $\Sigma$ at constant time. Their expression is naturally split in two pieces 


$$
\begin{aligned}
& Q(\xi)=q(\xi)+q_{0}(\xi) \\
& q(\xi)=-\int_{\partial \Sigma} \kappa_{D} \sqrt{-h} \epsilon_{i_{1} \ldots i_{2 n}}\left(\xi^{k} K_{k}^{i_{1}}\right)\left(\delta_{\left[j_{2} j_{3}\right]}^{\left[i_{2} i_{3}\right]}+2 \alpha(D-2)(D-3) \hat{R}_{j_{2} j_{3}}^{i_{2} i_{3}}\right) \\
& d x^{\dot{j}_{2}} d x^{j_{3}} d x^{i_{4}} \ldots d x^{i_{2 n}}+2 n c_{2 n} \sqrt{-h} \int_{0}^{1} d t \epsilon_{i_{1} \ldots i_{2 n}}\left(\xi^{k} K_{k}^{i_{1}}\right) \delta_{j_{2}}^{i_{2}} \\
& \left(\frac{1}{2} \hat{R}_{j_{3} j_{4}}^{i_{3} i_{4}}+\frac{t^{2}}{\ell_{e f f}^{2}} \delta_{j_{3}}^{i_{3}} \delta_{j_{4}}^{i_{4}}\right) \ldots\left(\frac{1}{2} \hat{R}_{j_{2 n-1} i_{2 n}}^{i_{2 n-1} i_{2 n}}+\frac{t^{2}}{\ell_{\text {eff }}^{2}} \delta_{j_{2 n-1}}^{i_{2 n-1}} \delta_{j_{2 n}}^{i_{2 n}}\right) d x^{\dot{j}_{2}} \ldots d x^{j_{2 n}}(13) \\
& q_{0}(\xi)=2 n c_{2 n} \int_{\partial \Sigma} \sqrt{-h} \int_{0}^{1} d t t \epsilon_{i_{1} \ldots i_{2 n}} \xi^{k}\left(\delta_{j_{2}}^{i_{2}} K_{k}^{i_{1}}+\delta_{k}^{i_{2}} K_{j_{2}}^{i_{1}}\right) \\
& \left(\frac{1}{2} R_{j_{3} j_{4}}^{i_{3} i_{4}}-t^{2} K_{j_{3}}^{i_{3}} K_{j_{4}}^{i_{4}}+\frac{t^{2}}{\ell_{e f f}^{2}} \delta_{j_{3}}^{i_{3}} \delta_{j_{4}}^{i_{4}}\right) \ldots \\
& \left(\frac{1}{2} R_{j_{2 n-1} j_{2 n}}^{i_{2 n-1} i_{2 n}}-t^{2} K_{j_{2 n-1}}^{i_{2 n n-1}} K_{j_{2 n}}^{i_{2 n}}+\frac{t^{2}}{\ell_{e f f}^{2}} \delta_{j_{2 n-1}}^{i_{2 n-1}} \delta_{j_{2 n}}^{i_{2 n}}\right) d x^{j_{2}} \ldots d x^{j_{2 n}} .
\end{aligned}
$$

It can be shown that $q(\xi)$-in any odd dimension- can be factorized by the l.h.s. of eq. (3), such that it identically vanishes for spacetimes that satisfy (3) globally. Then, $q_{0}(\xi)$ provides a tensorial formula for the vacuum energy for AAdS spacetimes.

The static black hole solution of EGB gravity is 16]

$$
d s^{2}=-\Delta^{2}(r) d \mathrm{t}^{2}+\frac{d r^{2}}{\Delta^{2}(r)}+r^{2} \gamma_{\underline{m}} \underline{n} d \theta^{\underline{m}} d \theta^{\underline{n}},
$$

where $\Delta^{2}(r)=k+\frac{r^{2}}{2(D-1)(D-2) \alpha^{*}}\left[1 \pm \sqrt{1+8 \Lambda \alpha^{*}+\frac{4(D-1)(D-2) \alpha^{*} \mu}{r^{D-1}}}\right]$, with $\mu$ appearing as an integration constant, and $\gamma_{\underline{m}} \underline{\underline{n}}$ $(\underline{m}, \underline{n}=1, \ldots, D-2)$ is the metric of the transversal section $\Sigma_{D-2}^{k}$ of constant curvature $k= \pm 1,0$. Solution (15) possesses an event horizon $r_{+}$, which is the largest solution to $\Delta^{2}\left(r_{+}\right)=0$. For the time-like Killing vector $\xi=\partial_{\mathrm{t}}$, the mass is given by

$$
\begin{aligned}
q\left(\partial_{\mathrm{t}}\right) & =M=\operatorname{vol}\left(\sum_{D-2}^{k}\right)(D-2) !\left(\Delta^{2}\right)^{\prime}\left\{\kappa _ { D } \left[r^{D-2}+\right.\right. \\
\left.\left.+2 \alpha(D-2)(D-3) r^{D-4}\left(k-\Delta^{2}\right)\right]+n c_{2 n} r \int_{0}^{1} d t\left(k-\Delta^{2}+\frac{t^{2} r^{2}}{\ell_{e f f}^{2}}\right)^{n-1}\right\}\left.\right|^{\infty} & \\
& =\frac{(D-2) \operatorname{vol}\left(\sum_{D-2}^{k}\right)}{16 \pi G_{D}} \mu,
\end{aligned}
$$

(a prime stands for $d / d r$ ), while the vacuum energy is

$$
\begin{gathered}
q_{0}\left(\partial_{\mathrm{t}}\right)=E_{0}=2 n c_{2 n}(D-2) ! \operatorname{vol}\left(\Sigma_{D-2}^{k}\right)\left(\Delta^{2}-\frac{r\left(\Delta^{2}\right)^{\prime}}{2}\right) \times \\
\left.\int_{0}^{1} d t t\left(k-t^{2} \Delta^{2}+\frac{t^{2} r^{2}}{\ell_{e f f}^{2}}\right)^{n-1}\right|^{\infty} \\
=(-k)^{n} \frac{\operatorname{vol}\left(\sum_{D-2}^{k}\right)}{8 \pi G_{D}} \ell_{e f f}^{2 n-2} \frac{(2 n-1) ! !^{2}}{(2 n) !}\left(1-\frac{2 \alpha}{\ell_{\text {eff }}^{2}}(D-2)(D-3)\right) \cdot(17)
\end{gathered}
$$

The orientation $\left(e^{1}, e^{0}, e^{\underline{m}}\right)$ is used throughout. Expression (17) specialized for $D=5$ agrees with the vacuum energy obtained by the surface counterterms method carried out in [17]. In the limit of vanishing Gauss-Bonnet coupling, formula (17) reduces to the vacuum energy for Schwarzschild-AdS found with Dirichlet counterterms [ 6$]$.
Black Hole Thermodynamics. The Euclidean period $\beta$ is defined as the inverse of black hole temperature $T$ such that in the Euclidean sector the solution (15) does not have a conical singularity at the horizon and it is given by $\beta=4 \pi /\left.\left(\Delta^{2}\right)^{\prime}\right|_{r_{+}}$. In the canonical ensemble, the Euclidean action $I^{E}=S-\beta \mathcal{E}$ defines the entropy $S$ and the thermodynamic energy $\mathcal{E}=-\frac{\partial I^{E}}{\partial \beta}$ of a black hole for a fixed surface gravity. The Euclidean bulk action is evaluated for a static black hole of the form (15) as

$$
\begin{aligned}
I_{b u l k}^{E}=-\kappa_{D}(D-2) ! & \operatorname{vol}\left(\sum_{D-2}^{k}\right) \beta\left\{( \Delta ^ { 2 } ) ^ { \prime } \left[r^{D-2}\right.\right. \\
& \left.\left.+2 \alpha(D-2)(D-3) r^{D-4}\left(k-\Delta^{2}\right)\right]\right\}\left.\right|_{r_{+}} ^{\infty},
\end{aligned}
$$

and the Euclidean boundary term as

$$
\begin{aligned}
\int_{\partial M} B_{2 n}^{E} & =-n(D-2) ! \operatorname{vol}\left(\sum_{D-2}^{k}\right) \beta\left[r\left(\Delta^{2}\right)^{\prime} \int_{0}^{1} d t\left(k-\Delta^{2}+\frac{t^{2} r^{2}}{\ell_{e f f}^{2}}\right)^{n-1}\right. \\
& \left.+2\left(\Delta^{2}-\frac{r\left(\Delta^{2}\right)^{\prime}}{2}\right) \int_{0}^{1} d t t\left(k-t^{2} \Delta^{2}+\frac{t^{2} r^{2}}{\ell_{e f f}^{2}}\right)^{n-1}\right]\left.\right|^{\infty} \cdot(19)
\end{aligned}
$$

The contribution of the bulk action $I_{b u l k}^{E}$ at radial infinity plus the Euclidean boundary term $c_{2 n} \int_{\partial M} B_{2 n}^{E}$-by virtue of eqns. (1617) -is equal to $-\beta\left(M+E_{0}\right)$. The finiteness of the charges ensures that the divergencies at $r=\infty$ of the bulk Euclidean action are exactly canceled by the ones coming from the boundary term. The thermodynamic energy definition

$$
\mathcal{E}=-\frac{\partial I_{2 n+1}^{E} / \partial r_{+}}{\partial \beta / \partial r_{+}}=M+E_{0},
$$

recovers the same result for the total energy as from the Noether charges defined above. Then, the entropy is the contribution of the Euclidean action from the horizon

$$
S=\frac{\operatorname{vol}\left(\Sigma_{D-2}^{k}\right) r_{+}^{D-2}}{4 G_{D}}\left[1+\frac{2 k \alpha(D-2)(D-3)}{r_{+}^{2}}\right],
$$

which agrees with the result of 17] obtained by regularizing the action with surface counterterms, and of [18] using the prescription of [19] at the horizon.

Even-dimensional case. For $D=2 n$ dimensions, the boundary term is given by the (maximal) $n$-th Chern form 20]

$$
\begin{array}{r}
B_{2 n-1}=n \int_{0}^{1} d t \epsilon_{A_{1} . . A_{2 n}} \theta^{A_{1} A_{2}}\left(\mathcal{R}^{A_{3} A_{4}}+t^{2} \theta_{C}^{A_{3}} \theta^{C A_{4}}\right) \ldots \\
\ldots\left(\mathcal{R}^{A_{2 n-1} A_{2 n}}+t^{2} \theta_{F}^{A_{2 n-1}} \theta^{F A_{2 n}}\right) \\
=2 n \sqrt{-h} \int_{0}^{1} d t \delta_{\left[i_{1} \ldots i_{2 n-1}\right]}^{\left[j_{1} \ldots j_{2 n-1}\right]} K_{j_{1}}^{i_{1}}\left(\frac{1}{2} R_{j_{2} j_{3}}^{i_{2} i_{3}}-t^{2} K_{j_{2}}^{i_{2}} K_{j_{3}}^{i_{3}}\right) \ldots \\
\ldots\left(\frac{1}{2} R_{j_{2 n-2} i_{2 n-1}}^{i_{2 n-2} i_{2 n-1}}-t^{2} K_{j_{2 n-2}}^{i_{2 n-2}} K_{j_{2 n-1}}^{i_{2 n-1}}\right) d^{2 n-1} x .(23)
\end{array}
$$

The on-shell variation of the action (2) is written as

$$
\begin{aligned}
& \delta I_{2 n}=\int_{\partial M} 2 \kappa_{D} \epsilon_{a_{1} \ldots a_{2 n-1}} \delta K^{a_{1}}\left(e^{a_{2}} e^{a_{3}}+\right. \\
& \left.\quad+2 \alpha(D-2)(D-3) \hat{\mathcal{R}}^{a_{2} a_{3}}\right) e^{a_{4}} \ldots e^{a_{2 n-1}} \\
& +2 n c_{2 n-1} \epsilon_{a_{1} \ldots a_{2 n-1}} \delta K^{a_{1}} \hat{\mathcal{R}}^{a_{2} a_{3}} \ldots \hat{\mathcal{R}}^{a_{2 n-2} a_{2 n-1}},
\end{aligned}
$$


and vanishes for AAdS spacetimes (3) with an appropriate choice of the coupling constant $c_{2 n-1}$

$$
c_{2 n-1}=(-1)^{n} \ell_{e f f}^{2 n-2} \frac{\kappa_{D}}{n}\left(1-\frac{2 \alpha}{\ell_{e f f}^{2}}(D-2)(D-3)\right) .
$$

The asymptotic condition (3) ensures a well-defined action principle for even-dimensional EGB gravity, that is the guiding line to achieve the finiteness of the conserved charges constructed by the Noether theorem

$$
\begin{gathered}
Q(\xi)=-\int_{\partial \Sigma} \kappa_{D} \sqrt{-h} \epsilon_{i_{1} \ldots i_{2 n-1}}\left(\xi^{k} K_{k}^{i_{1}}\right)\left(\delta_{\left[j_{2} j_{3}\right]}^{\left[i_{2} i_{3}\right]}+2 \alpha(D-2)(D-3) \hat{R}_{j_{2} j_{3}}^{i_{2} i_{3}}\right) \\
d x^{j_{2}} d x^{j_{3}} d x^{i_{4}} . . d x^{i_{2 n-1}} \\
+n \frac{c_{2 n-1}}{2^{n-2}} \sqrt{-h} \epsilon_{i_{1} \ldots i_{2 n-1}}\left(\xi^{k} K_{k}^{i_{1}}\right) \hat{R}_{j_{2} j_{3}}^{i_{2} i_{3}} \ldots \hat{R}_{j_{2 n-2} i_{2 n-1}}^{i_{2 n-2} i_{2 n-1}} d x^{j_{2}} . . d x^{j_{2 n-1}} \cdot(26)
\end{gathered}
$$

The mass for EGB-AdS black holes comes from the above formula for $\xi=\partial_{t}$, that is

$$
\begin{aligned}
& Q\left(\partial_{\mathrm{t}}\right)=M=\operatorname{vol}\left(\sum_{D-2}^{k}\right)(D-2) !\left(\Delta^{2}\right)^{\prime}\left\{\kappa _ { D } \left[r^{D-2}+\right.\right. \\
& \left.\left.+2 \alpha(D-2)(D-3) r^{D-4}\left(k-\Delta^{2}\right)\right]+n c_{2 n-1}\left(k-\Delta^{2}\right)^{n-1}\right\}\left.\right|^{\infty} \\
& \quad=\frac{(D-2) \operatorname{vol}\left(\sum_{D-2}^{k}\right)}{16 \pi G_{D}} \mu,
\end{aligned}
$$

expression that again agrees with the standard results in the literature 21]. The Euclidean continuation of the boundary term is now given by

$$
\int_{\partial M} B_{2 n-1}^{E}=-\left.n(D-2) ! \operatorname{vol}\left(\Sigma_{D-2}^{k}\right) \beta\left(\Delta^{2}\right)^{\prime}\left(k-\Delta^{2}\right)^{n-1}\right|^{\infty} .
$$

The total Euclidean action $I_{2 n}^{E}=I_{b u l k}^{E}+c_{2 n-1} \int_{\partial M} B_{2 n-1}^{E}$ at infinity corresponds to $-\beta M$, where $M$ is the Noether mass computed above. The same expression for the mass is obtained from the thermodynamic energy

$$
\mathcal{E}=-\frac{\partial I_{2 n}^{E} / \partial r_{+}}{\partial \beta / \partial r_{+}}=M
$$

For the entropy, the formula (21) is recovered for the $D=2 n$ dimensional case [18].

Conclusions. Unlike the usual methods to compute the conserved quantities and entropy in EGB gravity, here we have introduced for all dimensions the explicit form of the boundary terms that remove the divergencies in the energy definition and Euclidean action. We have also shown that in order to satisfy the first law of black hole thermodynamics in odd dimensions, the energy must appear as shifted by a vacuum energy respect to the mass obtained in background-substraction methods. The general formula for the energy of AdS vacuum might provide some insight on how gravity induces a corresponding CFT on the boundary.

In the standard regularization of AdS gravity, one can always add a counterterm that is a local functional of the boundary metric. It has been proposed [18] that this ambiguity might solve the problem of negative values of eq.(21), shifting the entropy by a constant. On the contrary, in the present approach, additional boundary terms would in general spoil the AAdS boundary conditions considered here. The only remaining freedom is the possibility of adding -in even dimensions- the Euler topological term $\mathcal{E}_{2 n}$ 15] instead of the Chern form (22). In that case, the entropy differs from the expression (21) by a constant related to the Euler characteristic $\chi(M)$ of the manifold, as noticed in 22] for EH-AdS gravity.

Acknowlegements The work of G.K. was supported by the European Commission Marie-Curie Fellowship under contract MEIF-CT-2004-501432. R.O. was supported by Fundação para a Ciência e Tecnologia (FCT) of the Ministry of Science, Portugal, through project POCTI/FNU/44648/2002.
[1] A.G. Riess et al., Astron. J. 116 (1998) 1009; S. Perlmutter et al., Astrophys. J. 517 (1999) 565; A.G. Riess et al., Astrophys. J. 607 (2004) 665; D.N. Spergel et al., astro-ph/0603499

[2] L. Randall and R. Sundrum, Phys. Rev. Lett. 83 (1999) 4690; P. Binetruy, C. Deffayet, U. Ellwanger and D. Langlois, Phys. Lett. B477 (2000) 285; G. Dvali, G. Gabadadze and M. Porrati, Phys. Lett. B485 (2000) 208; C. Deffayet, Phys. Lett. B502 (2001) 199.

[3] J. Maldacena, Adv. Theor. Math. Phys. 2 (1998) 231; Int. J. Th. Phys. 38 (1999) 1113; E. Witten, Adv. Theor. Math. Phys. 2 (1998) 253.

[4] V. Balasubramanian and P. Kraus, Comm. Math. Phys. 208 (1999) 413.

[5] M. Henningson and K. Skenderis, JHEP 9807 (1998) 023; S. de Haro, K. Skenderis and S. Solodukhin, Commun. Math. Phys. 217 (2001) 595.

[6] R. Emparan, C. Johnson and R. Myers, Phys. Rev. D60 (1999) 104001.

[7] C. Fefferman and C. Graham, "Conformal Invariants", in Elie Cartan et les Mathématiques d'aujourd'hui
(Astérisque, 1985) 95; C. Graham, math.DG/9909042 C. Graham and E. Witten, Nucl. Phys. B546 (1999) 52.

[8] B. Zwiebach, Phys. Lett. B156 (1985) 315.

[9] C. Germani and C. Sopuerta, Phys. Rev. Lett. 88 (2002) 231101; C. Charmousis and J. Dufaux, Class. Quant. Grav. 19 (2002) 4671.

[10] G. Kofinas, R. Maartens and E. Papantonopoulos, JHEP 0310 (2003) 066.

[11] S. Nojiri and S. Odintsov, Phys. Lett. B444 (1998) 92; Int. J. Mod. Phys. A 15 (2000) 413.

[12] A. Schwimmer and S. Theisen, JHEP 0310 (2003) 001.

[13] O. Miskovic and R. Olea, hep-th/0603092

[14] P. Mora, R. Olea, R. Troncoso and J. Zanelli, hep-th/0412046

[15] R. Aros, M. Contreras, R. Olea, R. Troncoso and J. Zanelli, Phys. Rev. Lett. 84 (2000) 1647; Phys. Rev. D62 (2000) 044002.

[16] D. Boulware and S. Deser, Phys. Rev. Lett. 55 (1985) 2656; J.T. Wheeler, Nucl. Phys. B268 (1986) 737; R. Cai, Phys. Rev. D65 (2002) 084014.

[17] M. Cvetič, S. Nojiri and S. Odintsov, Nucl. Phys. B628 
(2002) 295.

[18] T. Clunan, S. Ross and D. Smith, Class. Quant. Grav. 21 (2004) 3447.

[19] V. Iyer and R. Wald, Phys. Rev. D50 (1994) 846; R. Wald and A. Zoupas, Phys. Rev. D61 (2000) 084027.

[20] S. Chern, The Ann. of Math. 45 (1944) 747; 46 (1945)
674.

[21] S. Deser and B. Tekin, Phys. Rev. Lett. 89 (2002) 101101; Phys. Rev. D67 (2003) 084009; A. Padilla, Class. Quant. Grav. 20 (2003) 3129.

[22] R. Olea, JHEP 0506 (2005) 023. 